\title{
AB008. Prosthetic tracheal replacement using stented aortic matrices
}

\section{Emmanuel Martinod $^{1,2}$, Patrice Guiraudet ${ }^{1,2}$, Dana M. Radu ${ }^{1,2}$, Ana-Maria Santos Portela ${ }^{1}$, Marine Peretti ${ }^{1}$, Ilaria Onorati ${ }^{1,2}$, Olivia Freynet ${ }^{3}$, Yurdagül Uzunhan ${ }^{2,3}$, Kader Chouahnia ${ }^{4}$, Christophe Trésallet ${ }^{5}$, Sadek Beloucif ${ }^{6}$, Hélène Rouard ${ }^{7}$, Anne Fialaire-Legendre ${ }^{7}$, Morad Bensidhoum ${ }^{8}$, Hervé Petite ${ }^{8}$, Makoto Miyara ${ }^{9}$, Valérie Besnard $^{2}$, Carole Planès ${ }^{2,10}$, Eric Vicaut ${ }^{11}$}

${ }^{1}$ Chirurgie Thoracique et Vasculaire, Assistance Publique - Hôpitaux de Paris (AP-HP), Hôpitaux Universitaires Paris Seine-Saint-Denis, Hôpital Avicenne, Université Paris 13, Paris, France; ${ }^{2}$ Laboratoire Hypoxia and the Lung INSERM UMR 1272, Université Paris 13, Paris, France; ${ }^{3}$ Pulmonology, ${ }^{4}$ Oncology, Assistance Publique Hôpitaux de Paris (AP-HP), Hôpitaux Universitaires Paris SeineSaint-Denis, Hôpital Avicenne, Université Paris 13, Paris, France; ${ }^{5}$ Service de Chirurgie Générale Viscérale et Endocrinienne, Hôpital de la Pitié-Salpêtrière, Faculté de Médecine Pierre-et-Marie-Curie (Paris 6), Sorbonne Universités, Assistance publique - Hôpitaux de Paris (APHP), Paris, France; ${ }^{6}$ Anesthesiology, Assistance Publique - Hôpitaux de Paris (AP-HP), Hôpitaux Universitaires Paris Seine-Saint-Denis, Hôpital Avicenne, Université Paris 13, Paris, France; ${ }^{7}$ EFS Ile de France, Banque des Tissus, Créteil, France; ${ }^{8}$ B3OA UMR CNRS 7052, Université Paris Diderot, Sorbonne Paris Cité, CNRS, Paris, France; ${ }^{9}$ Sorbonne Université, Inserm, Centre d'Immunologie et des Maladies Infectieuses (CIMI-Paris), Paris, France; ${ }^{10}$ Physiology, Assistance Publique - Hôpitaux de Paris (AP-HP), Hôpitaux Universitaires Paris Seine-Saint-Denis, Hôpital Avicenne, Université Paris 13, Paris, France; ${ }^{11}$ Unité de Recherche Clinique, Hôpitaux Saint LouisLariboisière-Fernand Widal, Université Paris Diderot, Paris, France

Correspondence to: Prof. Emmanuel Martinod. APHP Hôpital Avicenne, 125 rue de Stalingrad, 93009 Bobigny Cedex, France.

Email: emmanuel.martinod@aphp.fr.

Abstract: Airway transplantation remains a great surgical and biological challenge. This is still an unsolved problem for patients in therapeutic impasse because of major tracheobronchial lesions requiring surgical resection and airway reconstruction. Schematically, 5 principal ways of research have been explored with the use of synthetic prostheses, airway bio-prostheses, tracheal allografts, various autologous substitutes and more recently bioengineered conduits. The lack of prospective human studies did not allow standardizing surgical approaches. In 1997, we initiated a research program on airway transplantation using stented aortic matrices in the Laboratory of the Alain Carpentier Foundation, Paris. A series of 7 preclinical animal studies using a sheep model showed that autologous and fresh or cryopreserved allogeneic aortic grafts could be valuable tracheal, carinal and bronchial substitutes. The regeneration of epithelium and de novo generation of cartilage were observed within the aortic matrices, thus allowing stent removal at a mean of 6 months. These favorable results led to the first human applications in patients with extensive tracheal diseases or complex tumors that would otherwise require pneumonectomies. Recently, we confirmed that airway bioengineering using cryopreserved aortic allografts was feasible for complex tracheal and bronchial reconstruction in 13 human cases (Martinod et al. Feasibility of bioengineered tracheal and bronchial reconstruction using stented aortic matrices. FAMA 2018;319:2212-22). Twenty patients were prospectively included in the TRACHEOBRONCART study. Thirteen patients underwent tracheal $(n=5)$, bronchial $(n=7)$, or carinal $(n=1)$ replacement. Airway transplantation was not performed in 7 patients because of medical contraindication $(n=1)$, unavoidable pneumonectomy $(\mathrm{n}=1)$, exploratory thoracotomy only $(n=2)$, and a lobectomy or bilobectomy was possible $(n=3)$. The overall 90 -day mortality rate was $5 \%$. There was no mortality at 90 days among patients who underwent tracheal or bronchial reconstruction. The only mortality was observed in the patient who had a massive stroke after carinal transplantation. There was no adverse event directly related to the innovative surgical technique. Stent removal was performed at a postoperative mean of 18.2 months. At a median follow-up of 3 years 11 months, 10 of the 13 patients were alive. Of these 10 patients, 8 breathed normally through newly formed airways after stent removal. Regeneration of epithelium and de novo generation of cartilage were observed within aortic matrices from recipient cells. We confirmed in human the possibility of epithelium regeneration, de novo cartilage generation and stent removal. Our previous work has shown that the $-80{ }^{\circ} \mathrm{C}$ cryopreserved aortic allografts contained viable cells at time of implantation, as assessed by their ability to migrate and proliferate from explants. They released a large panel of cytokines and growth factors and exerted 
significant chemoattractant and proangiogenic effects toward endothelial cells when assessed in vitro. Thus, cells from cryopreserved aortic allografts could have orchestrated the regenerative process by stimulating progenitor/stem cell homing and organ healing. The recipient body was used as a natural bioreactor and allowed in vivo airway tissue engineering. After approval by French Regulatory Authorities (Assistance Publique Hôpitaux de Paris DRCD and DAJ), we started the prospective observational study TRITON01 (TRacheobronchial bIoengineering using aorTic matrices for airway recONstruction) as a part of routine care for our center. We used the same innovative surgical approach as the previous protocol (NCT01331863, clinicaltrials.gov identifier) published by our group. Seven new patients with a therapeutic impasse have been added to the study since March 13, 2019. Our principal objectives are to include prospectively new patients with end-stage tracheobronchial diseases (TRITON 01 study); to compare airway transplantation using stented cryopreserved aortic allografts to standard therapy in patients with a major thyroid cancer invading the trachea (TRITON 02 study); to decipher the mechanisms of de novo cartilage regeneration and immunoregulation within transplanted aortic matrices; to optimize de novo cartilage regeneration using in vitro preconditioning of cryopreserved aortic allografts or in vivo local injection of mesenchymal stem cells; and finally to test new types of bioengineered and/or 3D printed aortic grafts with a commercial potential for a wider dissemination.

Keywords: Trachea; bronchi; surgery; transplantation; tissue regeneration

doi: 10.21037/shc.2019.AB008

Cite this abstract as: Martinod E, Guiraudet P, Radu DM, Portela AM, Peretti M, Onorati I, Freynet O, Uzunhan Y, Chouahnia K, Trésallet C, Beloucif S, Rouard H, FialaireLegendre A, Bensidhoum M, Petite H, Miyara M, Besnard V, Planès C, Vicaut E. Prosthetic tracheal replacement using stented aortic matrices. Shanghai Chest 2019;3:AB008. 